\title{
TRADUCIR E INTERPRETAR LA ORALIDAD
}

\author{
Cesáreo Calvo Rigual \\ Cesareo.Calvo@uv.es \\ Universitat de València - IULMA \\ Nicoletta Spinolo \\ Nicoletta.Spinolo@unibo.it \\ Università di Bologna (Forlì)
}

\section{Resumen}

Se introduce el concepto de oralidad en un sentido amplio, para pasar a continuación a su relación con la traducción, un campo relativamente reciente. En primer lugar se estudia el papel de la oralidad en la traducción audiovisual (donde es esencial el concepto de oralidad prefabricada) y en la traducción escrita (en particular en la literaria, la teatral y la de cómics), así como la importancia que ciertos elementos lingüísticos (marcadores del discurso, interjecciones y onomatopeyas) tienen en la reproducción de la oralidad. En segundo lugar se analiza la presencia del elemento oral en la interpretación, en la que lógicamente juega un papel esencial. Entre los numerosos aspectos que podrían estudiarse se examinan los relacionados con la prosodia (entonación y voz, fluidez y disfluencias) y la velocidad de elocución.

\begin{abstract}
"Translating and interpreting orality"

The concept of orality is introduced in a broad sense, and then is discussed in relation with translation, a relatively recent field. The first part of the paper analyses the role of orality in audiovisual translation (with the fundamental concept of prefabricated orality) and in written translation (especially for literature, theatre and comics), as well as the importance of certain linguistic elements (discourse markers, interjections and onomatopoeias) in reproducing orality. The second part of the paper, on the other hand, analyses the presence of the oral element in interpreting, where it obviously covers a pivotal role. Some of the many aspects that could be taken into account, and that are presented here are prosody (intonation and voice, fluency and disfluency) and speech rate.
\end{abstract}


Palabras clave: Oralidad. Traducción. Interpretación.

Key words: Orality. Translation. Interpreting.

Editorial article, received on September 19, 2015.

MonTI Special Issue 3 (2016: 9-32). ISSN 1889-4178 


\section{Introducción}

La oposición entre oralidad y escritura nace lógicamente cuando el ser humano idea formas de plasmar -en una pared, en una tablilla, etc.- lo que antes solo poseía forma oral. Este proceso, que duró siglos e incluso milenios, permitió al ser humano dejar una huella de sí mismo vívida y explícita. A pesar de la anterioridad de lo oral (y el ser imprescindible: una lengua existe aun sin escritura), la lingüística -con contadas excepciones- ha centrado su atención en la lengua escrita, hecho que se ha repetido en los estudios sobre la traducción, como veremos.

El concepto de oralidad ha dado lugar a aproximaciones muy diferentes desde muchas disciplinas. Abascal (2004) recoge tres posibles aproximaciones a la oposición oral-escrito: a) como canales de difusión, uno fónico y otro visual; b) como dos registros, siendo lo oral propio de la informalidad y lo escrito de la formalidad; y c) como dos modelos gramaticales diferentes, donde según algunos lo oral tendría una forma caótica y estaría sujeto a reglas diferentes: nadie sostiene hoy una idea semejante, gracias al avance de la Pragmática, que ha ofrecido descripciones detalladas de los mecanismos que regulan la oralidad (por ejemplo en Briz 1998 o Bazzanella 1994, para el español y el italiano respectivamente). Quedarían, por tanto, dos aproximaciones a dicha dicotomía, aunque la aplicación en exclusiva de una sola de ellas resulta excesivamente reduccionista, más aún si lo que esperamos es establecer una oposición que contemple solo dos tipos excluyentes. En realidad se trata de una oposición gradual (en un contínuum) entre cuyos extremos (lo "muy oral" y lo "muy escrito") se colocan numerosas manifestaciones lingüísticas que no pueden calificarse ni como completamente orales ni como completamente escritas: un discurso leído en el Parlamento europeo es a menudo la ejecución de un texto anteriormente escrito, un mensaje SMS o de Whatsapp escrito a una amiga es a menudo la transposición por escrito de un texto meramente oral. Esto nos llevaría a la conclusión de que lo escrito puro o lo oral puro son menos frecuentes de lo que podríamos pensar y que, en cambio, los tipos que podríamos llamar híbridos se dan de manera habitual. Teniendo presente lo anterior, Koch \& Oesterreicher (2007) postularon 
que lo realmente importante en una realización lingüística no es su soporte material (escrito u oral) sino su concepción, que produciría un contínuum en cuyos extremos se colocarían la inmediatez y la distancia comunicativa. Cada texto se colocaría en un lugar de ese contínuum en función de su concepción, y cerca de los extremos podremos encontrar tanto textos orales como escritos.

Los primeros Estudios de Traducción, de mediados del siglo XX (Hurtado 2001: 123), no mostraron un especial interés por la oralidad y se centraron en la traducción escrita. Solo algo más tarde dirigieron su atención hacia la traducción oral o interpretación (con los estudios de Seleskovitch en los años 60; cfr. Hurtado 2001: 80-81). Pero es mucho más tardío el interés por la traducción de los elementos típicos de la oralidad en la traducción escrita (fundamentalmente la literaria) o en la traducción audiovisual (TAV), que no llega hasta bien entrados los años 90. La mayoría de los estudios sobre el tema han venido de los estudios descriptivos de traducción, con alguna excepción, como el caso de Bandia (2011, 2015), el cual, desde los estudios postcoloniales, ha estudiado las manifestaciones de la oralidad en las sociedades primitivas sin escritura y los fenómenos de traducción que se dan en ellas al entrar en contacto con las culturas colonizadoras occidentales.

El interés actual por la traducción de la oralidad se refleja en un creciente número de publicaciones, como las siguientes, que citamos a título de ejemplo: Ballard (2001a), Brumme (2008a, 2008b, 2012), Gambier \& Lautenbacher (2010), Brumme \& Espunya (2012), San Vicente \& Morillas (2014), Bandia (2015). ${ }^{1}$

La oralidad no se presenta de igual manera ni tiene el mismo peso en todas las modalidades (en el sentido de Hurtado 2001: 69 ss.) de traducción, concretamente en la traducción escrita y en la TAV por un lado y la traducción oral (es decir, la interpretación) por otro. Esta oposición se basa, aparentemente, en que la interpretación es la traducción de una oralidad espontánea mientras que la traducción escrita y la audiovisual se enfrentan en cambio a una oralidad no espontánea. Aun siendo conscientes de que esta distinción no deja de ser engañosa, puesto que, como hemos visto antes, no siempre existe esta relación biunívoca entre tipo de oralidad y modalidad de traducción, nos basaremos en ella, pues resultará útil para profundizar en las cuestiones relacionadas con la traducción de la oralidad.

1. La bibliografía actual sobre el tema excede con mucho la posibilidad de ser recogida aquí en su totalidad; cuando un tema haya sido tratado en relación en varias combinaciones lingüísticas, daremos preferencia a la de italiano-español. 


\section{Traducción escrita y traducción audiovisual: la oralidad no espontánea}

Muchos textos escritos o audiovisuales contienen frases, palabras, etc., $\mathrm{u}$ otros elementos que pretenden emular la oralidad espontánea. Se trata, por ejemplo, de novelas u otras obras narrativas en las que se desea que los personajes hablen de manera creíble. En el caso del teatro nos encontramos, como para el resto de textos audiovisuales, ante una emulación total de la oralidad, puesto que se trata de escuchar la voz de personajes que para ser creíbles deben utilizar mecanismos característicos de la oralidad espontánea. En el planteamiento de Koch \& Oesterreicher (2007) este tipo de oralidad ocuparía a veces una posición intermedia y otras una más cercana a uno de los dos polos señalados, ya que en sí misma no constituye una modalidad de oralidad plenamente reconocible y establecida, sino que varía de un texto a otro, de unos géneros y autores a otros. De hecho, faltan estudios de alcance general ${ }^{2}$, que vayan más allá de los estudios de caso.

La terminología empleada para expresar este hecho es variada (Brumme 2008a: 8), no solo en función de los diferentes autores que la han tratado, sino también de cada tradición nacional y lingüística. En español encontramos una variedad de términos: oralidad fingida (Brumme 2008a, 2008b), oralidad prefabricada (Chaume 2001), junto a otros menos utilizados en los Estudios de Traducción, como oralidad literaria, oralidad simulada, etc.

Chaume (2001; Baños-Piñero \& Chaume 2009) ha sido pionero en el estudio de este importante aspecto en la TAV, en especial en el doblaje, donde los textos deben sonar naturales y creíbles, lo que no quiere decir imitar simiescamente la oralidad real, sino realizar una selección de rasgos fonéticos, morfológicos, sintácticos y léxico-semánticos de la lengua hablada para propiciar esa credibilidad: la diferencia consiste en que la frecuencia de esos rasgos en la TAV es menor que en la lengua hablada espontánea.

Desde el campo de la traducción literaria Schneider-Mizony (2010) parte del mismo presupuesto y llama a este tipo de oralidad "oralité de fiction". El traductor literario tiene dos opciones: traducir realizando una mímesis de la lengua oral o bien recurriendo a una oralidad convencional. La elección ha recaído en una u otra estrategia según la época, el tipo de texto, la intención del autor, el destinatario, etc. Distingue tres estrategias de oralización, que se combinarán con las opciones anteriores: a) la inclusión de informaciones pragmáticas (la gesticulación en el teatro, los deícticos, las marcas fáticas, etc.); b) la inclusión de marcas metalingüísticas (elementos tipográficos como

2. Quizá con la excepción de Romero-Fresco ((2012)), citado más adelante. 
guiones, comillas, etc.; de verbos introductores en la interacción comunicativa, etc.); y c) la adaptación de los elementos culturales de la oralidad. ${ }^{3}$

\subsection{La traducción audiovisual}

Romero Fresco (2012) analiza una cuestión directamente relacionada con la presencia de la oralidad prefabricada en la TAV, la (impresión) de naturalidad (entendida como "nativelike selection of expression in a given context", 2012: 199). Compara tres corpus de español (uno de lengua oral auténtica, otro de lengua oral prefabricada y otro de lengua oral prefabricada traducida) Aunque el estudio se centra en unos pocos marcadores del discurso, metodológicamente resulta de gran interés para evaluar la adecuación o no de la traducción de los elementos de la oralidad en un producto audiovisual.

Como señala Chaume (2004a), la TAV no es un tipo de traducción especializada, comparte con el resto de modalidades de traducción los mismos problemas y requiere estrategias similares, también en lo que respecta a la oralidad: son comunes, por tanto, los problemas relacionados con las variedades diatópicas, diastráticas o diafásicas, con las interjecciones y onomatopeyas, con los elementos culturales, el humor, las referencias intertextuales, etc. (Zabalbeaskoa 2008, 2011; Bernal Merino 2002). La diferencia estriba -como ya hemos dicho- en su menor frecuencia en la TAV y, además, en la existencia de dos factores específicos señalados por Zabalbeaskoa (2011): la escasez crónica de tiempo del que dispone el traductor y las constricciones debidas a los diferentes tipos de sincronía (sobre todo sincronía labial y la isocronía). Estas observaciones se refieren al doblaje, puesto que el subtitulado entraña problemas diferentes, ligados a la desaparición de casi toda la parte fónica del discurso (aunque sigue oyéndose el original) y a la limitación del espacio disponible donde colocar la traducción, lo que conlleva ciertas modificaciones, no siempre indoloras.

Aunque la teoría aconseja la conservación en las traducciones audiovisuales, en la medida de lo posible, de muchos elementos de la oralidad, en realidad se producen drásticas transformaciones que redundan al final en una falta de credibilidad (en el doblaje) fruto de la eliminación de dichos elementos (p. ej. los marcadores del discurso), de la reducción de la variedad lingüística

3. Debido a las limitaciones de espacio nos referiremos a los tipos de texto que han sido más estudiados, aunque no son, ni mucho menos, los únicos que contienen elementos de oralidad. Sin querer ser exhaustivos, podemos citar además, entre otros: documentos o textos históricos, declaraciones en juicios, publicidad, otros géneros narrativos tanto para adultos como para jóvenes y niños, documentales, videoclips y programas de televisión de todo tipo. 
del original a favor de una sola variedad neutra, estándar, en la traducción (Zabalbeaskoa 2008; Dolç \& Santamaria 1998) y de unas voces que no modulan adecuadamente.

\subsection{La traducción literaria}

Entre los géneros literarios, la oralidad prefabricada es especialmente frecuente en los géneros narrativos, tanto en los destinados a adultos (novela, narración breve, etc.) como entre los dirigidos a jóvenes y niños, aunque la cuestión de la oralidad ha sido estudiada sobre todo en la novela. Como señala Cadera (2001: 35), muchas novelas contemporáneas se caracterizan precisamente por el papel fundamental que en ellas ha adquirido la oralidad, construida con dos tipos de recursos: a) literarios (técnicas narrativas, recursos gráficos como las comillas, etc.); b) lingüísticos (uso de variedades lingüísticas y de otros elementos propios de la oralidad). Cadera defiende que ambos son imprescindibles y que por tanto se deben traducir, por lo que atender exclusivamente a los recursos lingüísticos, al puro contenido del texto, es un error. En una línea similar, Rosa (2015) defiende que lo que el traductor no debe perder de vista al traducir una novela con muchos diálogos y diferentes variedades diafásicas y diastráticas es el parámetro del prestigio, pues suele producirse una contraposición entre la variedad prestigiosa usada en la narración y otra menos prestigiosa usada en los diálogos. Por desgracia, muchos traductores tienden a eliminar esta importante diferencia, alterando algo tan fundamental para la caracterización de los personajes.

Son numerosos los estudios sobre la traducción de la oralidad de autores y obras literarias de muchas combinaciones lingüísticas. Limitándonos a la traducción del italiano al español o al catalán podemos citar los siguientes autores italianos: Gadda (Briguglia 2009), Pasolini (Briguglia 2009), Camilleri (Briguglia 2009; Caprara 2007).

\subsection{La traducción teatral}

La traducción teatral se ha estudiado a menudo como un subgénero de la traducción literaria, pero es evidente que existen diferencias importantes que aconsejan tratarlas por separado. Si bien existe una larga tradición de textos teatrales concebidos para ser leídos (para los que sirven las consideraciones expresadas en \$2.2.), la mayoría de ellos están concebidos para convertirse en lengua oral en escena, de nuevo bajo la modalidad de oralidad fingida.

La traducción de la oralidad de los textos dramatúrgicos se ha abordado desde dos perspectivas diferentes en Cebrián Alberola (2011): a) como 
aquello en lo que se apoya la representación; b) como imitación del discurso oral espontáneo. Ambas dan lugar a dos tipos de análisis diferenciados, aunque el segundo es sin duda el que interesa a los Estudios de Traducción. El estudio de obras clásicas -que siguen despertando interés en la actualidad: basta con citar el caso de Shakespeare- supone problemas particulares ligados a la distancia cronológica, tal como muestra Pujol (2011).

\subsection{La traducción de cómics}

En este género (que incluye numerosos tipos, incluyendo algunos recientes como la novela gráfica: cfr. el estudio de Rodríguez Abella en este volumen), como sucedía en la TAV, hay una estrecha dependencia entre los códigos visual y lingüístico: es, pues, una traducción subordinada (Hurtado 2001: 72). Los cómics se suelen presentar bajo forma de diálogo, que para ser creíble debe incluir marcas de oralidad (Delesse 2001: 321).

La oralidad es de nuevo prefabricada, aunque en este caso no siempre se persigue la imitación de la lengua espontánea, ya que son frecuentes los personajes que se expresan con un registro más bien elevado o de manera estereotipada: Corto Maltés, Capitán Trueno, Astérix, etc. El texto está situado en un espacio acotado (el de los globos o bocadillos) del que el traductor no puede salirse. Fuera de estos globos aparecen a menudo onomatopeyas o ideófonos (Muñoz-Calvo 2013, Zanettin 1998; Delesse 2001) integrados en el dibujo, que raramente va a poder modificar el traductor, lo que puede llevar a incongruencias debido a que estos elementos no son necesariamente universales. Carreras, Flores \& Provezza (2008: 17-18) señalan varios elementos del cómic que se deben tener en cuenta a la hora de traducir, entre los que destacan los derivados de la imitación de la oralidad: modismos, idiomatismos, coloquialismos, jerga juvenil, etc., e incluso un uso particular de los signos de puntuación, de los tipos y tamaños de letra o de las mayúsculas y minúsculas, que sirven para expresar estados de ánimo o intenciones de los personajes.

\subsection{La traducción de los rasgos de la oralidad en la traducción escrita y audiovisual}

Otro enfoque frecuente consiste en el estudio de ciertas clases de palabras en lugar de estudiar en su conjunto obras, autores o géneros. Hay una de esas clases que sobresale sobre las demás: los marcadores del discurso. Resulta lógico si pensamos que se trata de unos elementos fundamentales en la construcción de la conversación. La cuestión se ha abordado de dos maneras: los 
estudios de caso y los análisis contrastivos no centrados en obras concretas (como el de Solsona en este volumen).

Un problema que se señala en todos los estudios sobre la traducción de los marcadores (Biagini 2010; Calvo Rigual 2015; González 2012; Zamora \& Alessandro 2013) es su frecuente omisión al ser considerados superfluos, aunque a veces ello puede deberse a las constricciones del medio, como en el subtitulado (o en el doblaje por la sincronía labial). Según Chaume (2004b: 854) su omisión en el doblaje y en la subtitulación de películas podría estar en parte justificada porque a menudo las imágenes suplen las inferencias que vehiculan los marcadores, es decir, podrían considerarse redundantes respecto a la imagen (Biagini 2010: 21). Pero no son raros los casos en los que su omisión se debe a la impericia del traductor, que no comprende el sentido de un marcador (Calvo Rigual 2015). Varios autores que se han ocupado de la traducción de estos elementos (Aijmer, Foolen \& Simon-Vandenbergen 2006; Borreguero Zuloaga \& López Serena 2010) han fijado previamente sus características, que son fundamentalmente tres: su polifuncionalidad, su dependencia del contexto y lo escurridizo de su significado, lo que no quiere decir que no posean significado. Todo ello hace que sea muy difícil establecer equivalencias fijas o unívocas entre marcadores de diferentes lenguas (Bazzanella \& Morra 2000: 149-150; Portolés Lázaro 2002), incluso en casos de gran afinidad tipológica -como en el caso del español y del italiano-. Incluso marcadores que poseen una forma idéntica en dos lenguas resultan tener siempre un comportamiento divergente, al menos parte de sus funciones (Portolés Lázaro 2002: 152-156). En realidad, para traducir los marcadores el traductor ha de comprender su significado pragmático en la lengua fuente, buscando un equivalente que produzca el mismo efecto (Chaume 2004b: 844).

Otra categoría estudiada recurrentemente es la de las interjecciones, a la que se asocian las onomatopeyas. De todas ellas se ha ocupado Ballard (2001b), que considera que no se trata de simples sonidos que imitan la realidad, sino que poseen un significado, lo que las convierte en elementos susceptibles también de traducción. Entre otros estudios concretos sobre interjecciones señalamos Aja Sánchez (2011), Matamala (2007, 2008) o de Zamora \& Alessandro en este volumen.

\section{Oralidad e interpretación}

Definir la oralidad como una característica típica y fundamental de la interpretación, ya que la misma se define precisamente como una forma de traducción oral (Riccardi 1999), es seguramente cierto aunque, quizás, reductivo. En el caso de la interpretación, de hecho, la oralidad adquiere una multiplicidad 
de formas y representaciones que constituyen factores determinantes en la caracterización del discurso original, por un lado, y el discurso interpretado, por otro. Como afirma Stenzl (1983:40):

While in translation the message is conveyed entirely by graphic means, interpretation involves not only linguistic elements and what they convey, but also intonation, voice quality, changes in pitch and loudness, pauses and non-linguistic elements [...], which can all contribute to the message and may have to be verbalized by the interpreter.

La oralidad, sin embargo, no es solo una característica del texto original y del texto interpretado, es el medio a través del que viaja la prestación del intérprete, su herramienta de trabajo. Resulta fácil entender, pues, que el intérprete debe necesariamente tener un conocimiento profundo y completo de las propiedades de la oralidad, tanto para comprender las características del texto original, como para elaborar un texto meta adecuado y accesible: entonación (Collados Aís 1998, 2001, 2007), fluidez (Pradas Macías 2007), utilización de las pausas (Viaggio 1992), hesitaciones, velocidad de elocución, articulación, calidad de la voz (Iglesias Fernández 2007a, 2007b, 2013).

A partir de estas premisas, se entenderá la dificultad de la tarea de realizar una descripción panorámica de los estudios llevados a cabo hasta ahora sobre el tema. Sin ánimo de exhaustividad, por lo tanto, nos limitaremos en esta sección a presentar los principales temas tratados por los estudios de interpretación en las cuestiones más estrechamente relacionadas con la oralidad (voz, prosodia, fluidez, velocidad de elocución, etc.); dejaremos de lado, por razones de espacio, los relativos a aspectos lingüísticos (contenido, gramática, sintaxis, estilo, etc.), sin olvidar que estos últimos están siempre, indisoluble y primariamente conectados con las características de la lengua oral y, por consiguiente, con la interpretación y dependen, en muchos casos, de variables relacionadas con dicha modalidad expresiva. Esta restricción del ámbito tratado, motivada por cuestiones de espacio, obliga también a una restricción de los ámbitos de la interpretación tratados por dichos estudios: la mayoría de los autores presentados, de hecho, se centra fundamentalmente en el análisis de la interpretación de conferencias, mientras que son muy pocos los estudios que analizan la interpretación dialógica bajo este prisma.

Una contribución fundamental para el estudio de la oralidad en interpretación fue, a finales de los años 90, el nacimiento de los Corpus-based Interpreting Studies (véanse, entre otros, Shlesinger 1998; Russo, Bendazzoli, Sandrelli \& Spinolo 2012), que inauguraron una nueva fase de riguroso estudio empírico de material interpretado real, debidamente transcrito y anotado. Otra aportación fundamental para el estudio de la oralidad en interpretación 
fue la de Collados, Pradas, Stévaux \& García (2007), que contribuyeron a ratificar y definir la importancia de las características y del uso de la voz en la evaluación de la calidad de la interpretación simultánea, incluyendo, entre los parámetros a medir para analizar la calidad, la agradabilidad de la voz (Iglesias Fernández), la fluidez (Pradas Macías), la entonación (Collados Aís) y la dicción (Blasco Mayor \& García Becerra).

Entre los diferentes estudios realizados sobre oralidad basándose en corpus, resulta interesante el estudio de Russo (2014), que, trabajando con la combinación español-italiano, estudia los efectos de algunos rasgos críticos de la oralidad presentes en el texto original sobre el discurso interpretado, concluyendo que la producción del intérprete es necesaria y significativamente influenciada y guiada por fenómenos como la velocidad de elocución, la presencia de disfluencias, la falta de cohesión sintáctica, los paralelismos sintácticos y el uso de verbos y locuciones que indican la posición del hablante con respecto al público. Russo observa en la producción del intérprete fenómenos de simplificación de la sintaxis y omisión de la mayoría de los elementos con fuerte valor pragmático, además de numerosas operaciones de síntesis del mensaje original y de prosodia y énfasis, con el resultado de un texto meta más sintético, pero coherente y cohesionado.

Otro estudio original, en este caso de tipo cualitativo y con un enfoque holístico al estudio de la oralidad, es el de Anfuso \& Morelli (2014), que analizan un corpus de interpretación consecutiva español-italiano con el objetivo de identificar y analizar algunos de los rasgos típicos de la oralidad, estudiando además las estrategias empleadas por los intérpretes (estudiantes) para preservar, eliminar o reproducir dichos rasgos. Los resultados indican que, en la mayoría de los casos, los sujetos omiten los rasgos de la oralidad evidenciados en el discurso original y, cuando los mantienen, lo hacen modificándolos. Los autores no detectan, por otra parte, estrategias específicas empleadas por los intérpretes en la gestión de dichos elementos y lo atribuyen a la falta de experiencia de los sujetos.

\section{La prosodia en interpretación}

Como afirma Ahrens (2005: 1), la prosodia forma parte integrante de un texto producido oralmente, ya que sirve para estructurar el contínuum acústico producido por el orador y para atribuir mayor o menor prominencia a aquellas partes de texto que considera más o menos importantes. Los elementos prosódicos, pues, desempeñan un papel fundamental para determinar la comprensión de un texto oral por parte del receptor, y para vehicular las intenciones 
comunicativas del orador. Esto vale tanto para el lenguaje espontáneo como para la interpretación (Alexieva 1990; Ahrens 2005).

La mayor parte de los autores que se ha dedicado al estudio de las características prosódicas de la interpretación de conferencias están de acuerdo en afirmar que este aspecto no ha recibido, durante mucho tiempo, la necesaria atención de parte de los estudiosos de la interpretación, que se han concentrado más en otros aspectos (técnicas de trabajo, procesos neurolingüísticos, transmisión de contenidos). Aunque muchos de los Interpreting scholars (entre otros, Herbert 1952; Gerver, Longley, Long \& Lambert 1989) mencionan voz y prosodia como aspectos fundamentales de la prestación del intérprete y como elementos peculiares de la producción del texto interpretado (Shlesinger 1994), solo en tiempos más recientes las características prosódicas han sido analizadas y evaluadas como parámetros específicos. Collados Aís (2001: 105) subraya

la importancia que para la IS [interpretación simultánea] tiene la comunicación no verbal, teniendo en cuenta que la voz del intérprete de simultánea es el único vehículo que éste tiene a su disposición para la transmisión verbal y no verbal del discurso, y siendo la voz, por tanto, la que asume toda la responsabilidad de una interpretación de "calidad" y/o de "éxito".

\subsection{Entonación y voz}

Como afirman Pérez, Iglesias. Jiménez \& Blasco (2005) al definir algunos parámetros de evaluación de la calidad de la interpretación simultánea (2005: 1134), la voz constituye una herramienta de trabajo fundamental a disposición del intérprete para vehicular los contenidos del texto original de manera clara y eficaz; Collados Aís (2001) subraya incluso que el intérprete, como profesional de la comunicación, debería, sin perder nunca de vista contenidos y objetivos comunicativos del texto original, mejorar, cuando sea necesario, su presentación.

Uno de los primeros estudios sobre entonación y voz fue el de Darò (1990), que analizaba la frecuencia fundamental de la producción vocal de una intérprete profesional en cinco diferentes idiomas, suponiendo (y confirmando en los resultados) una posible correlación entre las variaciones de frecuencia de la voz y la mayor o menor seguridad del intérprete en un idioma u otro.

En 1994, Shlesinger estudia los efectos de la entonación del intérprete simultáneo sobre el público: analiza las producciones de ocho intérpretes profesionales, administrando a dos grupos de audiencia respectivamente un texto leído en voz alta y un texto interpretado, y midiendo su comprensión 
y retención de contenidos. Los resultados indican un mayor nivel de comprensión para el grupo de audiencia del discurso original con respecto al del discurso interpretado.

En la misma dirección, Collados Aís (1998) examina la incidencia de la entonación monótona en la evaluación de la calidad de la interpretación simultánea, concluyendo que una entonación monótona influye negativamente en la evaluación de la calidad por parte del público. En 2001 realiza un estudio experimental sobre el efecto de la entonación monótona en la recuperación de información por parte de quien escucha un discurso interpretado en simultánea. La conclusión principal es que, efectivamente, una entonación monótona parece influir negativamente en la recuperación de información por parte del usuario.

En 2005, Ahrens propone un posible enfoque al estudio de la prosodia en interpretación simultánea, analizando un corpus de textos originales y textos interpretados por profesionales, anotando pausas, unidades de información, patrones de acentuación y variaciones de tono como elementos constitutivos de las características prosódicas del texto interpretado.

Iglesias Fernández estudia la voz desde varios puntos de vista; con su estudio de 2007 (2007b), descubre que una interpretación precisa recibe una evaluación de calidad peor por parte del público si se presenta con voz desagradable, y que tono elevado y timbre nasal parecen indicar inseguridad e inexperiencia del intérprete, mientras que un tono más bajo y una mayor resonancia se asocian a una mayor credibilidad y experiencia. Descubre, además, que una voz desagradable influye en el juicio del público respecto a otras características prosódicas, como la entonación y la fluidez. Este resultado se ve fortalecido por un estudio de 2013 de la misma autora, que tiene el objetivo de sondear cuál es el concepto de "voz agradable" según el público; los resultados revelan que, efectivamente, el público tiende a extender el concepto de "voz de calidad" también a rasgos prosódicos como tono, entonación y fluencia.

Barbato (2014) estudia la influencia de la voz del intérprete jurídico en la percepción de credibilidad del mismo intérprete y del acusado por las partes y descubre, con su estudio-piloto, que el factor que más irrita a los usuarios de la interpretación es la entonación monótona, que se asocia a una personalidad insegura y poco creíble; se valoran positivamente, en cambio, velocidad y volumen moderados. 


\subsection{Fluidez y disfluencias}

La fluidez es uno de los aspectos de la oralidad que más atención ha recibido por parte de los estudiosos de interpretación. Con un estudio empírico, Pradas Macías (2007) indica que, en interpretación simultánea, la fluidez del texto meta puede influir en la percepción del público sobre otros parámetros, como una correcta transmisión del sentido y la precisión (en línea con los resultados de Collados Aís 1998 e Iglesias Fernández 2007a). También el estudio de Rennert (2010) indica una posible correlación entre la fluidez de un texto interpretado y la percepción de precisión por parte de los usuarios del mismo.

Una necesaria distinción, en el análisis de la fluidez en interpretación, se debe realizar entre la modalidad consecutiva y la simultánea:

Fluency in simultaneous is more subject to the quality of source speech delivery; in consecutive, the interpreter must achieve a good balance of careful listening and judicious use of notes, with ability to read notes at a glance and speaking skills coming to the fore during reformulation (Mead, 2005: 59).

En la interpretación consecutiva se centran, entre otros, los estudios realizados por Mead (2000, 2002 y 2005) y Cardoen (2012). Mead (2000) analiza el control de las pausas de estudiantes de interpretación, encontrando diferencias significativas en las prestaciones de estudiantes que trabajan hacia su lengua A (italiano) respecto a las de los mismos hacia su lengua B (inglés); estudia posteriormente las vacilaciones a través de un estudio empírico sobre textos interpretados en consecutiva por una muestra de intérpretes con distintos niveles de experiencia (Mead 2002) y, finalmente, propone un posible sistema metodológico para el estudio de la fluidez en interpretación consecutiva (Mead 2005). Por otra parte Cardoen (2012), con un estudio piloto llevado a cabo sobre la combinación español-neerlandés, analiza el impacto de la composición de las notas sobre la fluidez del intérprete consecutivo, comparando notas y texto-meta.

Mucho más amplio resulta, por el contrario, el panorama de los estudios sobre fluidez y disfluencias en interpretación simultánea, sobre la que, por razones de espacio, solo podremos proponer algunos ejemplos. Pöchhacker compara "slips and shifts" (1995: 74) en discursos espontáneos e interpretados, viendo solo parcialmente confirmada su hipótesis inicial, según la cual los discursos interpretados presentarían un número mayor de dichas disfluencias.

Dos posibles taxonomías de las disfluencias son las que proponen Tissi (2000) y Gósy (2007). Tissi (2000), en la combinación alemán-italiano, lleva a cabo un análisis descriptivo de las disfluencias presentes en los textos de 
origen y textos meta del corpus analizado, y las divide entre pausas vacías, alargamientos vocálicos y consonánticos, interrupciones, repeticiones, reestructuraciones y falsos arranques. Gósy (2007), en cambio, distingue en primer lugar entre disfluencias debidas a la incertidumbre y disfluencias-error; a la primera categoría pertenecen fenómenos como las vacilaciones, fillers, repeticiones, falsos arranques, alargamientos vocálicos y pausas internas en las palabras; a la segunda pertenecerían lapsus, errores gramaticales, contaminaciones, activación de falsas palabras, "tip of the tongue" y "ordering problems".

Cecot (2001) se centra en el estudio de las pausas analizando las prestaciones de once intérpretes en la combinación inglés-italiano, comparándolas con la percepción subjetiva de los profesionales sobre su prestación, y evidenciando numerosas incongruencias. Petite (2003, 2005), en cambio, se centra en las autocorrecciones, estudiando un corpus trilingüe (inglés/francés/ alemán) e identificando varias categorías de autocorrección en el proceso de automonitorización del intérprete. Trabajando con el par de idiomas españolitaliano, Bertozzi (2014) descubre que las disfluencias más frecuentes en el habla espontánea, como pausas llenas y vacías, alargamientos vocálicos, autocorrecciones, falsos arranques, son las más frecuentes también en el discurso interpretado. Plevoets \& Defrancq (en prensa), con un estudio basado en un corpus de interpretaciones simultáneas, estudian una tipología específica de disfluencias, las pausas llenas, con la intención de medir la carga cognitiva e informativa a la que se somete el intérprete, y correlacionando una mayor densidad de pausas llenas en el texto meta con una mayor densidad léxica en el texto original.

\subsection{Velocidad de elocución}

La velocidad de elocución es una característica del texto original que, comprensiblemente, puede influir en la prestación del profesional en todas las modalidades de interpretación. Se trata, de hecho, de una variable que muchos estudiosos (entre otros, Seleskovitch 1965, Alexieva 1990, Gile 1995 y Vuorikoski 2004) han evidenciado como problemática para el proceso de interpretación, ya que provoca en el intérprete una sobrecarga cognitiva y, por consiguiente, un texto meta de calidad inferior. A pesar de ello, numerosos autores han observado cómo, en realidad, una elevada velocidad de elocución no conlleva necesariamente, de por sí, una menor calidad del texto interpretado, ya que esta depende de una multiplicidad de factores concomitantes (contenidos, densidad léxica, expresividad, fluidez, etc.; Iglesias Fernández 2010). 
Trabajando sobre congresos de ámbito médico, también Galli (1990) observa que una mayor velocidad provoca un número mayor de omisiones e interpretaciones equivocadas de segmentos ambiguos, pero no influye, en general, en la precisión de la interpretación. El estudio de Pio (2003), en cambio, no analiza solo la equivalencia de contenido entre texto de origen y texto de llegada, sino que tiene además el objetivo de examinar el aspecto de la fluidez, observando, como Galli, que a un incremento de la velocidad corresponde, sobre todo, un incremento de los errores, especialmente las omisiones. Shlesinger (2003) se propone, por otra parte, estudiar el efecto de un elevado ritmo de elocución sobre la memoria de trabajo, parándose a observar su efecto en largas series de adjetivos concatenados. En este caso específico, la velocidad parece impactar positivamente en la prestación del intérprete, ya que los adjetivos concatenados se reproducen completamente, sobre todo, en los textos originales más rápidos. Iglesias Fernández (2010) estudia una muestra de discursos originales e interpretados del corpus cuatrilingüe ECIS, sometiéndolos a una comisión de seis evaluadores. También Iglesias Fernández, como Shlesinger, observa que una elevada velocidad de elocución no conlleva necesariamente una calidad inferior en la prestación del intérprete.

\section{Bibliografía}

AbASCAL, M. Dolores. (2004) La teoría de la oralidad. Málaga: Universidad de Málaga. Tesis doctoral inédita.

AHrens, Barbara. (2005) "Prosodic phenomena in simultaneous interpreting: A conceptual approach and its practical application." Interpreting 7:1, pp. 51-76.

AIJMER, Karin; Ad Foolen; Anne-Marie Simon-Vandenbergen. (2006) "Pragmatic markers in translation: a methodological proposal." In: Fischer, Kerstin (ed.) 2006. Approaches to Discourse Particles. Amsterdam: Elsevier, pp. 101-114.

AJA SÁNCHEZ, José Luis. (2011) "La interjección «aho» en las traducciones de los Racconti Romani. Algunas reflexiones sobre las estrategias traslativas de los rasgos lingüísticos regionales en el discurso oral". In: Romana García, María Luisa; José Manuel Sáenz Rotko \& Pilar Úcar Ventura (eds.) 2011. Traducción e interpretación: estudios, perspectivas y enseñanzas. Madrid: Universidad Pontificia de Comillas, pp. 17-36.

Alexieva, Bistra. (1990) "Creativity in simultaneous interpretation." Babel 36:1, pp. 1-6.

ANFUSO, Matteo \& Mara Morelli. (2014) "Oralidad y situación comunicativa en interpretación consecutiva: un estudio exploratorio". In: San Vicente, Félix 
\& Esther Morillas (eds.) 2014. Oralidad contrastiva español-italiano: aspectos gramaticales, discursivos y textuales. Cuadernos AISPI 4, pp. 129-147.

BALlARD, Michel. (2001a) Oralité et traduction. Arras: Artois Presses Université.

BALlARD, Michael (ed.) (2001b) "Onomatopée et traduction." In: Ballard, Michel

(ed.) 2001. Oralité et traduction. Arras: Artois Presses Université, pp. 13-42.

BANDiA, Paul F. (2011) "Orality and Translation." In: Gambier, Yves \& Luc van

Doorslaer (eds.) 2011. Handbook of Translation Studies. Amsterdam: John Benjamins, 2, pp. 108-112.

BANDiA, Paul F. (2015) "Introduction: Orality and translation." Translation Studies 8: 2, pp. 125-127.

BAÑOS-PiÑERO, Rocío \& Frederic Chaume. (2009) "Prefabricated Orality: A Challenge in Audiovisual Translation." inTRAlinea, Special Issue (The Translation of Dialects in Multimedia. M. Giorgio Marrano, G. Nadiani \& C. Rundle, eds.). Versión electrónica: http://www.intralinea.org/specials/ article/1714

BARBATO, Lucia. (2014) "La credibilidad de la voz del intérprete en la administración de justicia." Transfer 9:1-2, pp. 127-149.

Bazzanella, Carla \& Lucia Morra. (2000) "Discourse markers and the indeterminacy of translation.” In: Korzen, I. \& C. Marello (eds.) 2000. On Linguistic Aspects of Translation. Alessandria: Edizioni dell'Orso, pp. 149-157.

BAzzAnella, Carla. (1994) Le facce del parlare. Firenze: La Nuova Italia.

Bernal Merino, Miguel Angel. (2002) "Oralidad y traducción audiovisual." In: Bernal Merino, Miguel Ángel. 2002. La traducción audiovisual. Alicante: Universidad de Alicante, pp. 55-77.

Bertozzi, Michela. (2014) "Analisi delle disfluenze del discorso durante l'interpretazione simultanea da italiano a spagnolo." In: San Vicente, Félix \& Esther Morillas (eds.) 2014. Oralidad contrastiva español-italiano: aspectos gramaticales, discursivos y textuales. Cuadernos AISPI 4, pp. 149-164.

BiAgINI, Marta. (2010) "Les sous-titres en interaction : le cas des marqueurs discursifs dans des dialogues filmiques sous-titrés." Glottopol 15, pp. 18-33.

Blasco MaYor, María Jesús \& Olalla García Becerra. (2007) "La incidencia del parámetro dicción.” In: Collados Aís, Ángela; E. Macarena Pradas Macías; Elisabeth Stévaux \& Olalla García Becerra (eds.) 2007. La evaluación de la calidad en interpretación simultánea: parámetros de incidencia. Granada: Comares, pp. 175-194.

Borreguero ZuloagA, Margarita \& Araceli López Serena. (2011) "Marcadores discursivos, valores semánticos y articulación informativa del texto: el peligro del enfoque lexicocentrista." In: Aschenberg, H. \& Ó. Loureda Lamas (eds.) 2011. Marcadores del discurso: de la descripción a la definición. Fráncfort: Iberoamericana/Vervuert, pp. 169-210. 
BRiguglia, Caterina. (2009) La traducción de la variación lingüística en el catalán literario contemporáneo. Las traducciones de Pasolini, Gadda y Camilleri. Barcelona: Universitat Pompeu Fabra. Tesis doctoral inédita.

BRIZ, Antonio. (1998) El español coloquial en la conversación: esbozo de pragmalingüística. Barcelona: Ariel.

BRUMME, Jenny \& Anna Espunya (eds.) (2012) The translation of fictive dialogue. Amsterdam/New York: Rodopi.

BRUMME, Jenny (ed.) (2008a) La oralidad fingida: obras literarias: teatro, cómic y medios de comunicación. Madrid: Iberoamericana; Frankfurt: Vervuert, 2008.

BRUMME, Jenny (ed.) (2008b) La oralidad fingida: obras literarias: descripción y traducción. Madrid: Iberoamericana; Frankfurt: Vervuert.

BRUMME, Jenny. (2012) Traducir la voz ficticia. Berlin: De Gruyter.

CADERA, Susanne M. (2011) "Translating fictive dialogue in novels." In: Brumme, Jenny \& Anna Espunya (ed.) 2011. The translation of fictive dialogue. Amsterdam/New York: Rodopi, pp. 35-51.

Calvo Rigual, Cesáreo. (2015) "La traducción de los marcadores discursivos en la versión doblada española de la serie Il commissario Montalbano." Cuadernos de Filología Italiana 22, pp. 235-261.

CAPRARA, Giovanni (2007): Variación lingüistica y traducción: Andrea Camilleri en castellano. Málaga: Universidad de Málaga. Tesis doctoral inédita.

CARdoen, Hanne. (2012) "The effect of note-taking on target-text fluency." In: González Núñez, Gabriel; Yasmine Khaled \& Tanya Voinova (eds.) 2012. Emerging Research in Translation Studies: Selected Papers of the CETRA Research Summer School 2012. Electronic version: https://www.arts.kuleuven.be/cetra/ papers/files/cardoen [consultado el 23/11/2015].

Carreras i GoicoecheA, Maria; Estefanía Flores Acuña \& Monica Provezza. (2008) La traducción de cómics: Corto maltés, Lupo Alberto y Dylan Dog en español. Roma: Aracne.

Cebrián Alberola, Helena. (2011) "La traducción de la oralidad en los textos dramáticos. El caso de Cat on a Hot Tin Roof." Fórum de Recerca 16. Electronic version: http://repositori.uji.es/xmlui/bitstream/handle/10234/77258/ fr_2011_6_4.pdf?sequence=1

Chaume, Frederic. (2001) "La pretendida oralidad de los textos audiovisuales y sus implicaciones en traducción.” In: Agost, Rosa \& Frederic Chaume (eds.) 2001. La traducción en los medios audiovisuales. Castellón: Universitat Jaume I, pp. 77-88.

Chaume, Frederic. (2004a) "Film Studies and Translation Studies: Two Disciplines at Stake in Audiovisual Translation." Meta 49:1, pp. 12-25.

Chaume, Frederic. (2004b) "Discourse Markers in Audiovisual Translating." Meta 49:4, pp. 843-855. 
Collados Aís, Ángela; E. Macarena Pradas Macías; Elisabeth Stévaux \& Olalla García Becerra (eds.) (2007) La evaluación de la calidad en interpretación simultánea: parámetros de incidencia. Granada: Comares.

Collados Aís, Ángela. (1998) La evaluación de la calidad en la interpretación simultánea: la importancia de la comunicación no verbal. Granada: Comares.

Collados Aís, Ángela. (2001) "Efectos de la entonación monótona sobre la recuperación de la información en receptores de interpretación simultánea." Trans 5, pp.103-110.

Collados Aís, Ángela. (2007) "La incidencia del parámetro entonación." In: Collados Aís, Ángela; E. Macarena Pradas Macías; Elisabeth Stévaux \& Olalla García Becerra (eds.) 2007. La evaluación de la calidad en interpretación simultánea: parámetros de incidencia. Granada: Comares, pp. 159-174.

DARÒ, Valeria. (1990) "Voice frequency and simultaneous interpretation." The Interpreter's Newsletter 3/1990, pp. 88-92.

Delesse, Catherine. (2001): "Les dialogues de BD : une traduction de l'oral?" In: Ballard, Michel (ed.) 2001. Oralité et traduction. Arras: Artois Presses Université, pp. 321-340.

Dolç, Mavi \& Laura Santamaria. (1998) "La traducció de l'oralitat en el doblatge." Quaderns. Revista de traducció 2, pp. 97-105.

Galli, Cristina. (1990) "Simultaneous Interpretation in Medical Conferences: A Case-Study." In: Gran, Laura \& Christopher Taylor (eds.) 1990. Aspects of applied and experimental research on conference interpretation. Udine: Campanotto, pp. 61-82.

GAMBIER, Yves \& Olli Philippe Lautenbacher. (2010) "Oralité et écrit en traduction." Glottopol 15, pp. 5-17.

GerVer, David; Patricia Longley; John Long \& Sylvie Lambert. (1989) "Selection tests for trainee conference interpreters." Meta 34:4, pp. 724-735.

GILE, Daniel. (1995) Basic concepts and models for interpreter and translator training. Ámsterdam: John Benjamins.

GONZÁLEZ, Montserrat. (2012) "Pragmatic makers in translation.” In: Brumme, Jenny \& Anna Espunya (eds.). The translation of fictive dialogue. Amsterdam/ New York: Rodopi, pp. 217-232.

Gósy, Maria. (2007) "Disfluencies and Self-monitoring." Govor 24:2, pp. 91-110.

HERBERT, Jean. (1952) The Interpreter's Handbook: How to become a conference interpreter. Geneve: Georg.

IGLESIAS FERNÁNDEZ, Emilia. (2007a) "La incidencia del parámetro agradabilidad de la voz." In: Collados Aís, Ángela; E. Macarena Pradas Macías; Elisabeth Stévaux \& Olalla García Becerra (eds.) 2007. La evaluación de la calidad en interpretación simultánea: parámetros de incidencia. Granada: Comares, pp. 37-51. 
IGLESIAS FERNÁNDEZ, Emilia. (2007b) "La indefinición del parámetro «agradabilidad de la voz» y los estudios de calidad de la interpretación simultánea." In: Varela Salinas, María-José (ed.) 2007. La evaluación en los estudios de traducción e interpretación. Sevilla: Bienza, pp. 225-239.

IGLESIAS FERNÁNDEZ, Emilia. (2010) "Speaker fast tempo and its effects on interpreter performance: a pilot study of a multilingual interpreting corpus." The International Journal of Translation 22:1-2, pp. 205-228.

IGLESIAS FERNÁNDEZ, Emilia. (2013) "Understanding variability in interpreting quality assessment: user's sex and judgements for pleasant voice." In: Way, Catherine; Sonia Vandepitte; Reine Meylaerts \& Magdalena Bartłomiejczyk (eds.) 2013. Tracks and Treks in Translation Studies: selected papers from the EST Congress, Leuven 2010. Ámsterdam: John Benjamins, pp. 103-125.

Koch Peter \& Wulf Oesterreicher. (2007): Lengua hablada en la Romania: español, francés, italiano. Madrid: Gredos (trad. de Araceli López Serena; $1^{\mathrm{a}}$ ed. alemana: 1990).

Matamala, Anna. (2007) "The translation of oh in a corpus of dubbed sitcoms." Catalan Journal of Linguistics 6, pp. 117-136.

Matamala, Anna. (2008) "La oralidad en la ficción televisiva: análisis de las interjecciones de un corpus de comedias de situación originales y dobladas." In: Brumme, Jenny (ed.) 2008. La oralidad fingida: descripción y traducción. Teatro, cómic y medios de comunicación. Madrid: Iberoamericana; Frankfurt: Vervuert, pp. 81-94.

MEAD, Peter. (2000) "Control of pauses by trainee interpreters in their A and B languages." The Interpreters' Newsletter 10/200, pp. 89-102.

MEAD, Peter. (2002) "Exploring hesitation in consecutive interpreting." In: Garzone, Giuliana \& Maurizio Viezzi (eds.) 2002. Interpreting in the 21st Century: Challenges and Opportunities. Ámsterdam: John Benjamins, pp. 73-82.

MEAD, Peter. (2005) "Directionality and fluency: an experimental study of pausing in consecutive interpretation into English and Italian." Communication and Cognition. Monographies 38:1-2, pp. 127-146.

Muñoz-CAlvo, Micaela. (2013) “AYY!, ¡HUY!, ¡PAF!, ¡BOUM!, ¡ZAS!: Interjecciones, sonidos inarticulados y onomatopeyas en Astérix en Hispania y sus traducciones en las lenguas de la península ibérica." Transfer 8, pp. 117-152. Electronic version: http://www.raco.cat/index.php/Transfer/article/ view/269632

PÉREZ-LuZARDo DíAz, Jessica; Emilia Iglesias Fernández; Amparo Jiménez Yvars \& María Jesús Blasco. (2005) "Presentación y discusión de algunos parámetros de investigación en la evaluación de la calidad en interpretación simultánea." In: Romana García, María Luisa (ed.) 2005. II AIETI. Actas del II Congreso 
Internacional de la Asociación Ibérica de Estudios de Traducción e Interpretación. Madrid, 9-11 de febrero de 2005. Madrid: AIETI, pp. 1133-1154.

Petite, Christelle. (2003) "Repairs in Simultaneous Interpreting: Quality Improvement or Simple Error Corrections?" In: Collados Aís, Ángela; María M. Fernández Sánchez \& Daniel Gile (eds.) 2003. La evaluación de la calidad en interpretación: investigación. Granada, Comares, pp. 61-71.

PETITE, Christelle. (2005) "Evidence of repair mechanisms in simultaneous interpreting: A corpus-based analysis." Interpreting 7:1, pp. 27-49.

PIO, Sonia. (2003) "The relation between ST delivery rate and quality in simultaneous interpretation." The Interpreter's Newsletter 12, pp. 69-100.

Plevoets, Koen \& Bart Defrancq. (en prensa) "The effect of informational load on disfluencies in interpreting. A corpus-based regression analysis" Translation and Interpreting Studies 11:2.

PÖCHHACKER, Franz. (1995) "Slips and Shifts in Simultaneous Interpreting." In: Tommola, Jorma (ed.) 1995. Topics in Interpreting Research. Turku: The University of Turku, Centre for Translation and Interpreting, pp. 73-90.

PORTOLÉS LÁZARO, José. (2002) "Marcadores del discurso y traducción." In: García Palacios, Joaquín \& M Ma Teresa Fuentes Morán (eds.) 2002. Texto, terminología y traducción. Salamanca: Almar, pp. 145-167.

PradAS MACíAS, E. Macarena. (2007) "La incidencia del parámetro fluidez." In: Collados Aís, Ángela; E. Macarena Pradas Macías; Elisabeth Stévaux \& Olalla García Becerra (eds.) 2007. La evaluación de la calidad en interpretación simultánea: parámetros de incidencia. Granada: Comares, pp. 53-70.

Pujol, Dídac. (2011) "The translation of fictive dialogue in theatrical plays: some metalinguistic reflections.” In: Brumme, Jenny \& Anna Espunya (ed.) 2011. The translation of fictive dialogue. Amsterdam/New York: Rodopi, pp. 53-62.

RENNERT, Sylvi. (2010). "The impact of fluency on the subjective assessment of interpreting quality." The Interpreter's Newsletter 15/2010, pp. 101-115.

RICCARDI, Alessandra. (1999) "Interpretazione simultanea: strategie generali e specifiche." In: Falbo, Caterina; Mariachiara Russo \& Francesco Straniero Sergio (eds.) 1999. Interpretazione simultanea e consecutiva: problemi teorici e metodologie didattiche. Milan: Hoepli, pp. 161-174.

Romero Fresco, Pablo. (2012) "Dubbing dialogues... naturally. A pragmatic approach to the translation of transition markers in dubbing" MonTIMonografías de Traducción e Interpretación 4, 181-205.

RosA, Alexandra Assis. (2015) "Translating orality, recreating otherness." Translation Studies 8:2, pp. 209-225.

Russo, Mariachiara; Claudio Bendazzoli; Annalisa Sandrelli \& Nicoletta Spinolo. (2012) "The European Parliament Interpreting Corpus (EPIC): implementation and developments." In: Straniero Sergio, Franceso \& Caterina Falbo 
(eds.) 2012. Breaking Ground in Corpus-Based Interpreting Studies. Bern: Peter Lang, pp. 53-90.

Russo, Mariachiara. (2014) "Fenomeni dell'oralità critici per l'interpretazione simultanea: un'analisi contrastiva spagnolo-italiano basata sul corpus EPIC". In: San Vicente, Félix \& Esther Morillas (eds.) 2014. Oralidad contrastiva español-italiano: aspectos gramaticales, discursivos y textuales. Cuadernos AISPI 4, pp. 165-181.

SAN ViCENTE, Félix \& Esther Morillas (ed.) (2014) Cuadernos AISPI 4 (Monográfico sobre "Oralidad contrastiva español-italiano: aspectos gramaticales, discursivos y textuales").

SCHNEIDER-Mizony, Odile. (2010) "Traduire ou simuler l'oralité ?" Glottopol 15, pp. 80-95.

Seleskovitch, Danica. (1965) Colloque sur l'enseignement de l'interprétation. París: Asociación Internacional de Intérpretes de Conferencias.

SHLESINGER, Miriam. (1994) "Intonation in the production and perception of simultaneous interpretation." In: Lambert, Sylvie \& Barbara Moser-Mercer (eds.) 1994. Bridging the gap: Empirical research in simultaneous interpretation. Amsterdam: John Benjamins, pp. 225-236.

SHLESINGER, Miriam. (1998) "Corpus-Based Interpreting Studies as an Offshoot of Corpus-Based Translation Studies." Meta 43:4, pp. 486-493.

SHLESINGER, Miriam. (2003) "Effects of presentation rate on working memory in simultaneous interpreting." The Interpreter's Newsletter 12, pp. 37-49.

STENZL, Catherine. (1983) Simultaneous interpretation: groundwork towards a comprehensive model. Birkbek College, University of London. Tesis doctoral inédita.

TISSI, Benedetta. (2000) "Silent pauses and disfluencies in simultaneous interpretation: A descriptive anaysis." The Interpreter's Newsletter 10/2000, pp. 103-127.

ViAGGiO, Sergio. (1992) "Translators and interpreters: professionals or shoemakers?” In: Dollerup, Cay \& Anne Loddegaard (eds.) 1992. Teaching Translation and Interpreting: Training, Talent and Experience. Ámsterdam: John Benjamins, pp. 307-314.

VuORIKOSKI, Anna-Riitta. (2004) A voice of its citizens or a modern tower of Babel? The quality of interpreting as a function of political rhetoric in the European Parliament. Tampere: University of Tampere. Tesis doctoral inédita.

ZabalbeascoA, Patrick. (2008) "La credibilidad de los diálogos traducidos para audiovisuales." In: Brumme, Jenny (ed.) 2008. La oralidad fingida: obras literarias: descripción y traducción. Teatro, cómic y medios de comunicación. Madrid: Iberoamericana; Frankfurt: Vervuert, pp. 157-175. 
ZabalbeascoA, Patrick. (2011) "Translating dialogues in audiovisual fiction." In: Brumme, Jenny \& Anna Espunya (ed.) 2011. The translation of fictive dialogue. Amsterdam: Rodopi, pp. 63-78.

Zamora Muñoz, Pablo \& Arianna Alessandro. (2013) "Unidades fraseológicas periféricas, marcadores discursivos e interjecciones: consideraciones pragmáticas y discursivas en la traducción italiano/español.” In: Olza, Inés \& Elvira Manero Richard (eds.) 2013. Fraseopragmática. Berlin: Frank \& Timme, pp. 49-81.

ZANETTIN, Federico. (1998) "Fumetti e traduzione multimediale." IntTRAlinea 1. Electronic version: http://www.intralinea.org/archive/article/Fumetti_e_ traduzione_multimediale

\section{NOTAS BIOGRÁFICAS / BIONOTES}

Cesáreo Calvo Rigual es catedrático de Filología Italiana en la Universitat de València. Ha publicado estudios en los siguientes campos: lexicografía monolingüe (italiana) y bilingüe (italiano-español), lingüística contrastiva italiano-español, traducción audiovisual e historia de la traducción de obras italianas al español y al catalán. En este último campo ha contribuido a la creación y mantenimiento del Proyecto Boscán (Catálogo de las Traducciones Españolas de Obras Italianas (hasta 1939). Ha traducido al español obras de Pietro Aretino y de Giovanni della Casa además de "La veneciana" de anónimo veneciano del siglo XVI. Es autor, junto a Anna Giordano, del Diccionario italiano Herder (1995, 2005 y 2011).

NiCOLETTA SPINOLO es doctora en Traduzione, Interpretazione e Interculturalidad por la Universidad de Bolonia (Forlì, Departamento de Interpretación y Traducción DIT), docente de interpretación en la misma Universidad e intérprete profesional. Sus principales intereses de investigación son la interpretación simultánea del lenguje figurado, la didáctica de la interpretación dialógica y de conferencias y la interpretación a distancia.

Cesáreo Calvo Rigual is full Professor of Italian Philology at the Universitat de València. He has published studies in the following fields: monolingual (Italian) and bilingual (Italian-Spanish) lexicography, Italian-Spanish contrastive linguistics, audiovisual translation and history of the translation of Italian works into Spanish and Catalan. Lately, he has contributed to the setting up and production of the Proyecto Boscán (Catalogue of the Spanish Translation of Italian Works (until 1939). He translated into Spanish works by Pietro Aretino and Giovanni della Casa, as well as "La venexiana" by the 
anonymous venetian of the $16^{\text {th }}$ century. He is author, together with Anna Giordano, of the Diccionario italiano Herder (1995, 2005 y 2011).

Nicoletta SPINOlo holds a PhD in Translation, Interpreting and Intercultural Studies from the University of Bologna (Forlì, Department of Interpreting and Translation DIT), teaches interpreting at the same University and is a practicing conference interpreter. Her main research interests are the simultaneous interpreting of figurative language, dialogue and conference interpreter training and remote interpreting. 